\title{
Estrategias de prevención y vigilancia sobre el uso de sustancias ilícitas y su impacto en los costos de accidentes en conductores de carga pesada en México
}

\author{
Eduardo Puente-Rodríguez ${ }^{1}$ \\ Sandra Cristina Pillon²
}

\begin{abstract}
Las colisiones de tránsito son uno de los grandes problemas de Salud Pública en el mundo, los impactos afectan áreas de la salud, económica y social. Los conductores de carga pesada fueron el objeto de este estudio. Se tuvo por objetivo identificar los costos de accidentes después de aplicar estrategias de control de consumo de sustancias lícitas e ilícitas en conductores de transporte de carga pesada. Se realizó un estudio correlacional retrospectivo de 2004 a 2006. La población a la que se aplicó el programa disminuyó el número de accidentes en $15 \%$, y el costo por daños por colisión en $24 \%$. Se concluye que estudios de esta naturaleza son importantes para implantación de programas orientados a modificar la conducta cuidado y seguimiento de la salud de los conductores profesionales.

Descriptores: Prevención Primaria; Accidentes de Transito; Costos y Análisis de Costo; Bebidas Alcohólicas.
\end{abstract}

\footnotetext{
1 Postgrado de Salud Pública, Profesor, Facultad de Salud Pública y Nutrición, Universidad Autónoma de Nuevo Léon, Nuevo Léon, México. E-mail: e_puerod@hotmail.com.

2 Enfermera, Doctora en Enfermería. Profesor Asociado, Escola de Enfermagem de Ribeirão Preto, Universidade de São Paulo, Centro Colaborador de la OMS para el Desarrollo de la Investigación en Enfermería, SP, Brasil. E-mail: pillon@eerp.usp.br.
}

Correspondencia:

Sandra Cristina Pillon

Universidade de São Paulo. Escola de Enfermagem de Ribeirão Preto

Departamento de Enfermagem Psiquiátrica e Ciências Humanas

Av. dos Bandeirantes, 3900

Bairro: Monte Alegre

CEP: 14040-902 Ribeirão Preto, SP, Brasil

E-mail: pillon@eerp.usp.br 


\section{Estratégia de prevenção e vigilância sobre o consumo de sustâncias ilícitas e os custos dos acidentes em motoristas de carga pesada, no México}

Os acidentes de trânsito constituem um dos maiores problemas de saúde pública no mundo, e seus impactos afetam as áreas de saúde, econômica e social. O motorista de carga pesada é o objeto do presente estudo. O objetivo foi identificar os custos de acidentes ao aplicar estratégias de controle, frente ao uso de drogas lícitas e ilícitas, em motoristas de carga pesada. Como metodologia deste estudo foi usada a pesquisa retrospectiva correlacional, conduzida de 2004 a 2006. Com base no censo, na população participante do programa houve diminuição do número de acidentes de $15 \%$ e dos custos por danos causados por colisão de $24 \%$. Conclui-se que estudos dessa natureza são importantes para implantação de programas direcionados à modificação de comportamentos e monitorização do cuidado de saúde a motoristas profissionais.

Descritores: Prevenção Primária; Acidentes de Trânsito; Custos e Análise de Custos; Bebidas Alcoólicas

\section{Prevention and Monitoring Strategies on the Use of Illicit Substances and Accidents and Costs in Heavy Load Drivers in Mexico}

Road crashes are one of the largest public health problems in the world. Impacts affect Health, Economic and Social areas. This study focuses on heavy load drivers. The goal is to identify the costs of accidents to implement control strategies for licit and illicit substance consumption in heavy load drivers. Method: a retrospective correlation study was conducted, covering the period from 2004 to 2006. In the population covered by the program, the number of accidents decreased by $15 \%$ and cost for damage by collision by $24 \%$. Conclusion: This type of studies are of great value and can be used as a basis for implementing programs designed to modify behavior and carefully monitor the health of professional drivers.

Descriptors: Primary Prevention; Accidents, Traffic; Costs and Cost Analysis; Alcoholic Beverages.

\section{Introducción}

En el mundo una gran parte de las relaciones comerciales y económicas dependen de los medios de trasporte terrestre para trasladar las materias primas, productos y subproductos de la mayoría de las actividades de un país.

Los conductores que trabajan por largos períodos, poseen un estilo de vida y conductas de riesgo como la ingesta de alcohol durante su actividad de trabajo, ingesta de sustancias ilícitas para realiza y soportar por largas jornadas; otras características son: mala alimentación, sedentarismo, tabaquismo, y pocas horas de sueño, característica que frecuentemente los pone en riesgo de sufrir colisiones de tránsito(1).
Los estudios sobre los accidentes de tráfico en México son escasos, la prevención y el control se iniciaron hace recientemente y poco se sabe acerca del comportamiento del conductor, de las condiciones de seguridad de las carreteras y vehículos, de los costos humanos y ambientales, del uso de vehículos de motor y de las consecuencias derivadas de estas colisiones traumáticas.

Los datos del informe mundial sobre prevención de accidentes de tráfico presenta altas tasas de mortalidad y morbilidad que se producen en las carreteras, las que han generado pérdidas incalculables e inaceptables para la salud pública en general, social y económica; así, el 
informe mundial recomienda adoptar algunas medidas preventivas para los distintos países. Se estima que cada año 1,2 millones de personas mueren y más de 50 millones son mutiladas en las carreteras(2).

Se estima que los costos económicos derivados de estos daños son de tal magnitud que afectan los indicadores económicos de un país como es el Producto Interno Bruto (PIB) en el orden de 1 a $2 \%{ }^{(2)}$.

Según estimaciones moderadas, el costo mundial de los traumatismos por accidentes de tráfico asciende aproximadamente a US\$520.000 millones anuales. EI costo en los países en vías de desarrollo es de 65.000 millones de dólares que es más de lo que reciben los países en desarrollo en ayuda para el desarrollo(3). El porcentaje que se atribuye como promedio, a los países desarrollados es de un $2 \%$ de su PIB, y en países en vías de desarrollo entre un 1 y $2 \%$ de su PIB. Las colisiones con resultados fatales representaron cerca de $20 \%$ de ese costo, mientras que las colisiones de tránsito con lesionados ascendieron a casi dos tercios del costo total de los accidentes ${ }^{(4)}$.

En México y de acuerdo a la Secretaría de Comunicaciones y Transporte (SCT) los costos, en millones de pesos, por daños materiales referidos al año de 2004 fueron de 1.248 , en 2005 de 1.303 , y en 2006 de 1.407 , mostrando un aumento de $12,70 \%$ de 2004 a $2006^{(5)}$.

Es de destacar que no sólo en México sino en muchos países, la conducción en estado de ebriedad o bajo la influencia de alcohol está prohibida por ley y las violaciones terminan en sanciones.

Se sabe que el consumo de alcohol aumenta el riesgo de colisiones que causan la muerte o lesiones graves, sin embargo es difícil hacer comparaciones entre los diferentes estudios debido a las grandes variaciones que existen de un país para otro, en lo que se refiere a los límites legales, impuestos por ley, de alcohol en la sangre; por ejemplo, citamos algunos de los diferentes valores establecidos en América Latina: en Brasil - 0,6 $\mathrm{g} / \mathrm{l}$, en Argentina 0,5 g / I, en El Salvador, 1,0 g / I; en México - 0,8 g / l; existen países que ni siquiera existen normas ${ }^{(6)}$.

Los países que adoptaron una política restrictiva para controlar el consumo de alcohol entre los conductores, se encuentra reevaluando sus límites, ya que parece existir una tendencia a disminuir los niveles permitidos, por ejemplo, en Australia, la disminución de 0,8 para $0,5 \mathrm{~g} / \mathrm{l}$ se asoció con una disminución de $6 \%$ de los accidentes ${ }^{(7)}$.

Existen límites de consumo de alcohol y riesgo al conducir determinados desde $1964^{(8)}$.

\section{Accidentes de tránsito y trabajo}

Las colisiones en la vía pública en el continente europeo, incluidas las personas que trabajan, representan un porcentaje aún mayor de las muertes vinculadas con la actividad laboral, alrededor de $41 \%$ en $1999^{(9)}$. En los países en vías de desarrollo los datos sobre colisiones en la vía pública relacionados con el trabajo son escasos.

En los Estados Unidos, un promedio de 2.100 trabajadores murieron anualmente porcausa de colisiones en la vía pública, entre los años de 1992 a 2001. De acuerdo al Censo de Lesiones Ocupacionales Mortales, Oficina de Estadísticas Laborales, los accidentes viales ocuparon el primer lugar con el $22 \%$ de las muertes en el trabajo, y corresponde a algo más de $3 \%$ del total de muertes por choques en la vía pública. En el 2001 la Administración Nacional para la Seguridad del Tráfico de Carreteras señaló que 4,2 millones de trabajadores de vehículos automotores sufrieron accidentes, siendo $73 \%$ camioneros. Los accidentes viales son la causa principal de muerte de los trabajadores del transporte. En el año 2000, las pérdidas en los salarios y beneficios para las víctimas de choques (ocupacionales o no) ascendieron a 61.000 millones de pesos. Las pérdidas por las ausencias de los empleados al trabajo ascendieron a 4.600 millones de $\operatorname{pesos}^{(10)}$.

El Instituto Mexicano del Transporte ha señalado que las muertes en el transporte automotor (colisiones de tránsito) en México representan un tasa de riesgo de 13 a 15 por cada 100.000 , esto lo ubica dentro de los países que poseen valores elevados en este indicador ${ }^{(11)}$ La Secretaría de Comunicación y Trasporte de México $(\mathrm{SCT})^{(5)}$ reportó que por cada 100 accidentes de carretera en 1996 ocurrieron 57,3 lesionados; en el año de 2006 se presentaron el índice fue de 114,3 por cada 100 accidentes, lo que representan un $100 \%$ de aumento. En 1996 el número de muertos evolucionó de 8,3 para 16,9 muertos por cada 100 accidentes. Los datos informados por la SCT de México(10) respecto a los accidentes en carreteras del país en 2004, presentaron 30.668 accidentes con 31.274 lesionados y 4.603 muertos. En 2005 los números señalan que hubo 29.463 accidentes con 32.275 lesionados y 4.710 muertos. En el año 2006 se presentaron 29.078 con 33.130 lesionados y 5.014 personas muertas. De acuerdo a cifras de la SCT por cada 100 accidentes de carretera se presentaron 57,3 en 1996 a 114,3 en 2006, mostrando un aumento de casi el doble de lesionados; en el número de muertos, su evolución fue de 8,3 en 1996 para 16,9 por cada 100 accidentes; la institución no se refirió específicamente al tipo de transporte ${ }^{(5)}$. 
Las estadísticas de organismos de Canadá, Estados Unidos de Norteamérica y México, presentan en los registros publicados sobre accidentes ocurridos en carretera, que envuelven vehículos de trasporte de carga pesada, que $8 \%$ de los conductores mueren por causa de esos accidentes ${ }^{(12)}$. La SCT se refiere a evaluaciones médicas y exámenes para detección de drogas ilícitas; informando que en 2001 se realizaron 327.500 y en 2006 solamente 106.400 , lo que representa una disminución del $67 \%$; no fueron publicados los resultados de dichas evaluaciones, tampoco el número de pruebas positivas y el tipo de droga detectada(5).

\section{Programas de prevención}

La OMS en conjunto con la Organización International del Trabajo establece cuales pueden ser las pautas para un plan de control de consumo de sustancias en los centros de trabajo por medio de la publicación de tratamiento de cuestiones relacionadas con alcohol y drogas en el lugar de trabajo(13). El Centro de Controle de Enfermedades señala algunas estrategias de este tipo ${ }^{(10)}$.

En México la SCT ha establecido el funcionamiento del Comité de Prevención de Accidentes de Carretera y Vialidad que trabaja para formular un Programa de Seguridad Vial para las Carreteras Federales de México(11,14). Entre sus iniciativas están: control de Alcohol, Drogas; Control de la Exposición a Fatiga; Prevención de Accidentes; Modificación de la conducta, y Control de lesiones; y Manejo de lesionados.

El objetivo del estudio es identificar los resultados de los costos de accidentes al aplicar estrategias de control de consumo de sustancias lícitas e ilícitas en conductores de transporte de carga pesado (remolques) por medio de un Programa de salud y seguridad en una empresa.

\section{Metodología}

Se realizó un estudio retrospectivo de abordaje cuantitativo de corte trasversal en los años 2004 a 2006. El estudio es de tipo encuesta y se aplicó en dos grupos de trabajadores operadores del trasporte de carga pesada en México.

Población de Conductores de unidades de Carga pesada (remolques)

Población 1: 57.728 en 2004 a 60.615 en 2006. Conductores de carga pesada registrados en la SCT de México y colisiones de tránsito en carretera donde participaron unidades de carga pesada que no están en un programa de control de consumo de sustancias psicoactivas.

Población 2: 1.607 en 2004 a 2.576 en 2006. Conductores de empresa de transporte de carga pesada con distribución en México y colisiones de tránsito en carretera que han implementado programas y estrategias en el control de consumo de sustancias lícitas e ilícitas.

\section{Recolección de datos}

Secretaría de Comunicaciones y Transporte de México (SCT) y Empresa

Recolección directa de información estadística publicada por la SCT y el Instituto Mexicano del Trasporte (IMT) sobre conductores, vehículos, accidentes, lesiones, muertes, costos y evaluaciones médicas y el consumo sustancias lícitas e ilícitas, identificando si la empresa responsable del accidente había documentado y aplicado un programa de control de consumo de sustancias ilícitas.

Análisis de estadísticas, de la empresa de conductores, vehículos, colisiones, lesiones, muertes, costos y evaluaciones médicas y el consumo sustancias lícitas e ilícitas, donde se ha documentado y aplicado un programa de control de consumo de sustancias psicoactivas.

El programa implementado por la empresa fue compuesto de los siguientes elementos: Política de conductas saludables, Gestión y asignación de recursos para el programa. Operación del programa: prevención del consumo de sustancia lícita e ilícita, capacitación en efectos de sustancias lícitas e ilícitas, evaluación (física y toxicológica) médica anual de pre-operación y en operaciones en carretera. Seguimiento médico y apoyo a problemas de salud en el consumo de sustancias lícitas e ilícitas. Participación de la familia en la capacitación.

\section{Procedimiento}

Se realizó un análisis comparativo de los datos estadísticos entre las 2 poblaciones estudiadas.

Se obtuvo información sobre: la estadística de los años 2004 a 2006 de las poblaciones de conductores de carga pesada; el grupo SCT sin un programa de prevención y vigilancia en el consumo de sustancias ilícitas y sobre el grupo de conductores de empresa que utilizó el programa mencionado; número de vehículos de carga pesada en México y en la empresa: la aplicación de estrategias de control del consumo de sustancias lícitas e 
ilícitas; comportamiento de la población de conductores de carga pesada en México y el número registrado en la empresa; evaluaciones medicas a conductores de carga pesada; y, relación con costos por accidentes de tránsito.

\section{Resultados}

Los resultados del estudio comparativo de las poblaciones, en relación a la aplicación de Programa de prevención de consumo de drogas se presentan de forma separada y por elemento (vehículos, accidentes, lesiones y costos).

Tabla 1 - Comparativo de conductores y vehículos de carga pesada 2004 a 2006, México. 2008

\begin{tabular}{lcclcc}
\hline & \multicolumn{2}{c}{ Conductores } & & \multicolumn{2}{c}{ Vehículos } \\
\cline { 2 - 3 } \cline { 5 - 6 } & $\mathbf{N}$ & $\%$ & & $\mathbf{N}$ & $\%$ \\
\hline SCT & 57.728 a 60.615 & 5 & & 268.725 a 280.085 & 11 \\
Empresa & 1.607 a 2.576 & 60 & & 1.140 a 1.828 & 60 \\
\hline
\end{tabular}

Se observa en la Tabla 1 que los índices de conductores de carga pesada son altos en la SCT como en la empresa, observando que en esta última existe un aumento al mismo ritmo tanto en conductores como en vehículos de carga.

En la SCT la distribución es un conductor por cada 4,6 unidades. En la empresa se tiene una distribución de 1,4 conductores por cada unidad, datos no presentados en la tabla.

Tabla 2 - Comparativo de colisiones y transporte de carga pesada de 2004 a 2006, México. 2008

\begin{tabular}{lccccc}
\hline & \multicolumn{2}{c}{ Colisiones totales } & & \multicolumn{2}{c}{$\begin{array}{c}\text { Accidente por cada } 100 \\
\text { vehículos }\end{array}$} \\
\cline { 2 - 3 } \cline { 5 - 6 } & $\mathbf{N}$ & $\%$ & & $\mathbf{N}$ & $\%$ \\
\hline SCT & 30.668 a 29.078 & 5,18 & & 19,16 a 16,29 & 15 \\
Empresa & 64 a 54 & 15,6 & & 5,61 a 2,95 & 47 \\
\hline
\end{tabular}

El comportamiento de las colisiones es bajo en el número total de Colisiones de tránsito en los grupos estudiados. Se observa que los conductores de la SCT presentan una relación mayor de riesgo de sufrir accidente con respecto a los conductores de la Empresa, presentado en la Tabla 2.
Tabla 3 - Comparativo de las pruebas toxicológicas de 2004 a 2006, México. 2008

\begin{tabular}{|c|c|c|c|}
\hline & \multicolumn{2}{|c|}{$\begin{array}{l}\text { Evaluaciones } \\
\text { toxicológicas }\end{array}$} & \multirow{2}{*}{$\begin{array}{c}\text { Evaluaciones } \\
\text { toxicológicas por } \\
\text { conductor al año } \\
\mathrm{N}\end{array}$} \\
\hline & $\mathbf{N}$ & $\%$ & \\
\hline SCT & 170.200 a 106.400 & 37,48 & 1,2 a 0,9 \\
\hline Empresa & 456 a 5.647 & 12,38 & 4 a 3,1 \\
\hline
\end{tabular}

El número de pruebas toxicológicas en el total de conductores registrados en la SCT es menor como se muestra en la Tabla 3. En conductores de carga pesada en la SCT disminuyó de 2,94 para 1,7 evaluaciones por año. La comparación con las aplicadas en la empresa es de 1,7 / 2,19 evaluaciones por conductor, datos no presentados en tablas.

Tabla 4 - Comparativo de Costos de 2004 a 2006, México. 2008

\begin{tabular}{lccccc}
\hline & \multicolumn{2}{c}{$\begin{array}{c}\text { Costos en millones de } \\
\text { pesos }\end{array}$} & & \multicolumn{2}{c}{$\begin{array}{c}\text { Costos en miles de pesos } \\
\text { por accidentes }\end{array}$} \\
\cline { 2 - 3 } \cline { 5 - 6 } & $\mathbf{N}$ & $\%$ & & $\mathbf{N}$ & $\%$ \\
\hline SCT & 1.248 a 1.407 & 12,71 & & 40.703 a 48.388 & 18,88 \\
Empresa & 25.5 a 16.2 & 36,41 & & 398.609 a 300.389 & 24,64 \\
\hline
\end{tabular}

En la SCT el costo total presentó aumento tomando en cuenta todo tipo de accidentes. En la empresa el costo promedio por cada accidente disminuyó aproximadamente 98.220 pesos, presentado en la Tabla 4.

\section{Discusión}

Este estudio permitió evaluar y comparar por primera vez en México la situación en que se encuentran las instituciones incluidas en análisis de colisiones y costos con respecto a la aplicación de un programa de prevención y vigilancia en el consumo de drogas.

La estadística internacional señala que el número de accidentes (colisiones) presenta una tendencia a elevarse hasta $60 \%$ hasta el año de 2020. La Organización Mundial de la Salud señala que en los países más desarrollados y que están trabajando en forma intensa se espera una disminución de aproximadamente $2 \%$ anual, de los accidentes para llegar a un total de 30\% entre 2004 a $2020^{(10)}$.

Esto es cercano a lo informado por la SCT, la cual señala una disminución de $5 \%$ en el estudio (Tabla 1 ). 
El comportamiento de los accidentes en la empresa presenta una disminución de $15 \%$ de sus accidentes en los tres años mencionados (Tabla 2).

En el análisis de las colisiones se observó en los resultados una disminución de tres veces en la población dentro del programa de vigilancia con respecto a los registrados ante la SCT.

En México, en conductores del programa de prevención y vigilancia, el promedio en los tres años de análisis registró que 3,77 de cada 100 vehículos de carga sufrieron una colisión

En los EUA(10) el $33 \%$ de los accidentados en carretera son trabajadores del transporte. En el presente estudio, los trabajadores del programa, apenas $2 \%$ sufrieron colisiones en carretera.

En la Tabla 3 se observa una disminución de la evaluación toxicológica, pero, las medida de control son generales y no hay información cuando las autoridades refieran a cuantos conductores, de carga pesada, se les realizó una prueba de sustancia ilícita o aquellos que al tener una colisión dieron positivo para dicha prueba.

El programa de prevención y vigilancia establece que todos los operadores deben realizar tres exámenes al año. Las acciones de la SCT sobre la capacidad y conocimiento de los conductores de carga pesada, al exigirles que aprueben un examen y ser certificados por una institución especializada en este tema, denota avances en el sistema de administrar esta área de trabajo. Todos los operadores del programa tienen su licencia vigente como conductores por la SCT.

El comportamiento en costos en la SCT es claro, a pesar de la disminución del número de accidentes, los costo presentan un aumento de $12,71 \%$ en los tres años (Tabla 4). En 2005 la SCT calculó los costos de colisiones en 1.303 millones y en los vehículo de carga pesada alrededor de 635 millones de dólares, esto representó el $53,7 \%$ de los costos totales. Con un costo de 442.715 pesos por accidente. En la empresa el costo disminuyo de 398.000 pesos a 300.000 con una disminución de 24,64\% por cada colisión. Algunos autores refieren beneficios mayores cuando se aplican programas de prevención en el consumo de drogas ${ }^{(15-16)}$.

El abuso de alcohol es un problemas de salud que en todos los países está creciendo rápidamente. Los factores sociales y conductuales del estilo de vida como: sedentarismo, estrés, abuso de sustancias, accidentes de tráfico, y violencia, son amenazas a la salud en diferentes grupos de la población. Es un problema de salud que no respeta fronteras geográficas. Por lo cual, se necesitan nuevos enfoques, sobre todo para comprender mejor la manera de realizar intervenciones para reducir el consumo de drogas y su impacto, lo que debe incluir la reducción de costos y la promoción de estilos de vida saludables y calidad de vida(17).

\section{Limitaciones del estudio}

Cuando se realizó el presente estudio no fueron encontrados trabajos relacionados con el análisis del tema de programas de prevención y vigilancia en el consumo de drogas en conductores de México, tampoco específicamente sobre conductores de transporte de carga pesada. No existe disponible información de programas preventivos y de vigilancia del consumo de sustancias ilícitas registrado por las empresas con actividad en el transporte carga pesada.

Los informes estadísticos varían entre los años, evidenciando una falta de estabilidad en la información; la SCT por parte del gobierno establece mecanismos para evaluar a los conductores de carga pesada por medio de la aplicación de exámenes de conocimiento y certificación médica de buena salud para expedir una licencia de conductor, siendo esta una medida adecuada para registrar y controlar a las personas que realizan esta actividad, pero los datos señalan que existe un número excesivo de unidades registradas en comparación a los conductores autorizados.

\section{Conclusiones}

Las estrategias aplicadas actualmente en los conductores de carga pesada reflejan que cumplen con la finalidad de disminuir la frecuencia de los accidentes, pero no la gravedad de acuerdo con el costo de las colisiones en el autotransporte, incluyendo los de carga pesada.

Los costos presentan una tendencia a elevarse, lo que puede tener variables a ser estudiadas y así establecer si existen otros factores que intervengan en este indicador.

Se concluye que la aplicación de programas de prevención y vigilancia del consumo de sustancias ilícitas y la participación del conductor tomando consciencia de que su conducta influye en disminuir el riesgo de accidentes y por lo tanto de lesionarse o de de morir por colisiones de tránsito, pueden tener como efecto una disminución en el número y costo de las colisiones en el transporte de carga pesada en México. Es necesario involucrar a las empresas en el cuidado de la salud y seguridad del los conductores con la participación de las partes involucradas como son: gobierno, empresa, trabajadores y familia. 
Los beneficios esperados al aplicar programas de control en los conductores de carga pesada pueden representar un ahorro del $25 \%$ de los costos de los accidentes, también un riesgo menor de sufrir accidentes.

El análisis de los datos, sobre los accidentes y costos en conductores de carga pesada de las poblaciones estudiadas, demuestra que los costos económicos en las poblaciones que están bajo un programa de vigilancia del consumo de drogas influyen positivamente en este indicador.

Debido al alto costo social, económico y de salud que causan las colisiones de transporte de carga pesada, es de suma importancia desarrollar otros estudios sobre costos y efectos de aplicación de medidas de prevención y vigilancia de sustancias ilícitas en los conductores de carga pesada.

\section{Agradecimientos}

Agradecemos a la Comisión Interamericana para el Control del Abuso de Drogas/CICAD de la Secretaria de Seguridad Multidimensional/SSM de la Organización de los Estados Americanos/OEA, la Secretaria Nacional de Políticas sobre Drogas/SENAD do Gabinete de Seguridad Institucional/Brasil, la Escuela de Enfermería de Ribeirao Preto de la Universidad de Sao Paulo y Centro Colaborador de la Organización Mundial de la Salud para el Desarrollo de la Investigación en Enfermería, la población representada en los estudios de investigación, bien como a las autoridades de las universidades representadas por los participantes del Programa En-Line de Especialización en Investigación sobre el Fenómeno de las Drogas, periodos 2006, 2007, 2008 y 2009.

\section{Referencias}

1. Domingos JBC, Pillon SC. O uso de alcool entre motoristas no interior do Estado de São Paulo. Rev Enferm UERJ. 2007:15(3):393-9.

2. Peden M, Scurfield R, Sleet D, Mohan D, Hyder AA, Jarawan E, et al. The World Report on Road Traffic Injury Prevention. Geneva (Swz): World Health Organization; 2004.

3. Organización Mundial de la Salud (OMS). Folleto Oficial de Enlace Día Mundial de la Salud. Departamento de Prevención de los Traumatismos y la Violencia Ginebra Suiza. 2004. [acesso em 17 jun 2009]. Disponible en: www.who.int/world-health-day/es
4. Costo de los accidentes viales en Australia, Reporte 102. Oficina de Economía del Transporte, Canberra, Australia, 2000. [acesso em 20 mai 2009]. Disponible en: http://www.infrastructure.gov.au/roads/safety/ road_fatality_statistics/index.aspx

5. Secretaría de Comunicaciones y Transporte de México Anuarios Estadísticos y Operación 2004 a 2006. 2009. [acesso em 12 set 2009]. Disponible en: http://www. sct.gob.mx

6. Organización Mundial de la Salud. Informe Mundial sobre Prevención de los traumatismos causados por el Tránsito. Resumen. Ginebra; 2004.

7. Guppy A. At what blood alcohol concentration should drink-driving be illegal? Br Med J. 1994;(308):1055-6.

8. Krüger HP, Kazenwadel J, Vollrath M. Grand Rapids Effects Revisited: Accidents, Alcohol and Risk. [internet]. Proceedings 13 - 18 Aug 1995; Adelaide, Australia (AUS): 13th International Conference on Alcohol, Drugs and Traffic Safety; [acesso en 3 nov 2008]. 564 p. Disponible en: http://www.druglibrary.org/schaffer/ Misc/driving/s9p2.htm

9. National Highway Traffic Safety Administration. Traffic safety facts 2001: a compilation of motor vehicle crash data from the Fatality Analysis Reporting System and the General Estimates System. National Center for Statistics and Analysis. Washington, DC, 2002

10. Centro para el Control y Prevención de Enfermedades. Accidentes viales relacionados con el trabajo: estrategias de prevención para los empleadores. Publicación no. 137. Center for Disease Control and Prevention. USA Marzo 2004. [acesso en 03 nov 2008]. Disponible en: http:// www.cdc.gov/spanish/niosh/docs/pdfs/2004-136Sp.pdf 11. Díaz AM, Grajeda EFM. Aspectos Básicos para formular programas de seguridad vial para carreteras federales. Guía Técnica. N.178. Secretaria de Comunicación y Transporte / Instituto Mexicano del Transporte. Disponible en http://www.imt.mx/Espanol/ Publicaciones/pubtec/pt178.pdf

12. Estadísticas de Transporte de América del Norte. Base de Datos 2006. [internet]. [acesso en 03 nov 2008]. Disponible en: http://nats.sct.gob.mx/nats/sys/ tables.jsp? $\mathrm{i}=2 \& \mathrm{id}=12$

13. Organizacion Mundial del Trabajo (OIT). Tratamiento de cuestiones relacionadas con alcohol y las drogas en el lugar de trabajo. Repertorio de recomendaciones practicas de la OIT. Ginebra; 1996. 71 p.

14. Secretaría de Comunicaciones y Transporte de México. 
Acuerdo para crear el Comité nacional de prevención de accidentes en carretera y vialidad. Diario Oficial de la Federación México Distrito Federal. [periódico na internet]. 10 out 2000. [acesso 04 jul 2009]. Disponible en: http://www.sct.gob.mx/fileadmin/normatividad/ transporte_terrestre/24creaci\%d3n\%20\%20del\%20 conaprea.pdf

15. Roberts S, York J. Design, Development and Evaluation of Driver Wellness Programs. Federal Motor Carrier Safety Administration Office of Research and Technology. 1997. [acesso 03 jul 2009]. Disponible en: http://www.fmcsa.dot.gov/documents/wellness-2.pdf 16. National Highway Traffic Safety Administration. The Economic Burden of Traffic Crashes on Employers Costs by State and Industry and by Alcohol and Restraint Use. Cost. U.S. 2002. [acesso 19 set 2009]. Disponible en: http://www.nhtsa.dot.gov/people/injury/airbags/ economicburden/images/Traffic\%20crashes.pdf

17. Pillon SC, Luis MA. Modelos explicativos para o uso de álcool e drogas e a prática da enfermagem. Rev. Latino-Am. Enfermagem. 2004;12(4):676-82. 